\title{
Performance of an Adaptive PSO Partial Parallel Interference Canceller for CDMA
}

\section{Communication Systems}

\author{
Chia-Hsin Cheng* \\ The Department of \\ Electrical \\ Engineering \\ National Formosa \\ University \\ Yunlin County, \\ Taiwan \\ *chcheng@nfu.edu.tw
}

\author{
Hsi-Chou Hsu \\ The Department of \\ Computer and \\ Communication \\ Engineering, \\ National Pingtung \\ Institute of \\ Commerce \\ Pingtung City, \\ Taiwan
}

\author{
Yung-Fa Huang \\ The Department of \\ Information and \\ Communication \\ Engineering \\ Chaoyang \\ University of \\ Technology \\ Taichung County, \\ Taiwan
}

\author{
Jyh-Horng Wen \\ The Department of \\ Electrical \\ Engineering \\ Tung Hai University \\ Taichung City, \\ Taiwan
}

\author{
Ling-Cheng Hsu \\ The Department of \\ Information and \\ Communication \\ Engineering \\ Chaoyang \\ University of \\ Technology \\ Taichung County, \\ Taiwan
}

\begin{abstract}
In this paper, we propose an adaptive particle swarm optimization (APSO) based partial parallel interference cancellation scheme for direct-sequence code-decision multiple access (CDMA) communication system over frequency selective fading channels. However, the partial cancellation tries to reduce the cancellation error in parallel interference cancellation schemes due to the wrong interference estimations in the early stages. Thus, in order to reduce the computational complexity and improve the performacne of the CDMA system, particle swarm optimization (PSO), which has the ability of solution to complex combinatorial optimization problem, is employed in searching the optimal weight for multiple access interference cancellation. Experiments have been carried out on the method can improved on the multiuser detection in CDMA system, the simulation results show that APSO detector can achieve the global optimization in fast convergence rate, and also show that the proposed detector is obviously superior to convenentional receiver and multiuser detectors based on some optimum algorithm in terms of the multiple access interfere and the mitigation of near-far effect.
\end{abstract}

Keywords CDMA, partial parallel interference cancellation, partial cancellation weight, PSO.

\section{INTRODUCTION}

The DS-CDMA cellular communication system is one of the favorite candidates for $3^{\text {rd }}$ generation radio cellular communication systems with its potentially high system capacity [1].The two main shortcomings of direct-sequence code-decision multiple access (CDMA) systems, multiple access interference (MAI) and the near-far problem, degrade the performance. The multiuser detector, the mainstream research for CDMA receivers, which attempts to eliminate MAIs and the near-far problem simultaneously, has been accepted as a capacity improving technique and received much significant attention recently [2-6]. Unfortunately, in DS-CDMA communication system with large number of users, the employment of optimum multiuser [2] detection becomes difficult due to the large computational complexity involved. To overcome this computational complexity problem, many researchers have proposed linear suboptimal multiuser detectors with lower complexity and much better performance than the conventional matched filter detector [2],[4], and references therein. Among these suboptimal multiuser detectors, the linear PIC and SIC have been further analyzed using a matrix-algebraic approach in [5]. The parallel interference cancellation (PIC) scheme simultaneously removes the interference from each user's received signal [6]. However, at heavy system load, the conventional multistage PIC scheme, which attempts to completely cancel the MAIs, suffers performance degradations due to the poor cancellations, which are brought about by the relatively high error-rate of bit decisions in the preceding stage. Hence, the partial cancellation proposed by Divsalar et al. [7], which chooses not to cancel the entire amount of estimated multiuser interference, rather is a better policy than the complete PIC. However, even the optimal cancellation weight in a PPIC scheme is still unable to fully account for the bit-decision error in the previous stage.

The partial cancellation weight (PCW) of the PPIC scheme is a fraction of one, which reflects the reliability of the tentative decision. When perfect power control scheme is exerted, the bit error rate of each user should be kept the same and all the PCWs are certainly the same in one stage to reflect the same reliability of interference. Therefore, the partial cancellation weight (PCW) in a PPIC scheme can be updated by the least mean square (LMS) algorithm [8], which has been found to be superior to the CPIC and the constant weight PPIC $[9,10]$. Unfortunately, the high convergent speed and small steady state misadjustment error can not be improved simultaneously in the adaptive array when using LMS algorithm. Moreover, when the system load is heavy, the adaptation of the PCW becomes unreliable due to the low convergence of the LMS algorithm and the increasing mean-square error (MSE) degrades the performance. The investigation on the statistics of adaptation weight based on the LMS algorithm has been done in previous works $[9,10]$ and then an adaptive full PIC (AFPIC) scheme has been proposed to outperform the normalised LMS-based PPIC scheme for flat fading channels. The performance of the AFPIC scheme gets boosted over the 
LMS-PPIC at heavy system load as described in [9]. However, under heavy system load, the estimated signal based on the proposed bit-inversion procedure may not be absolutely reliable. Moreover, Divsalar[7], Wen.[11] and Hsieh [15], these PCW are determined by trial and error; thus, dynamically adjusting the weightings can be costly. Other methods for optimizing these factors have also been proposed [13-16]. In [16], for example, this PCW are adjusted using the normalized leased mean-square adaptitation algorithm, and the performance is sensitive to the value of the adaptation step size. Therefore, in this paper, a low -complexity normalized PSO-MSE algorithm is employed in searching for the optimal PCWs. On top of this, the products of the partial cancellation weights and the hard decisions made in the previous stage are used in regenerating the interference. Our simulation results show that the proposed technique can provide a superior performance over the convention PIC, the PPIC, AFPPIC, APPIC, and the the BI-LMS detectors.

The paper is organized as follows. The system model of DS-CDMA systems is described briefly in Section II. In Section III, the effectiveness of the partial cancellation weight of the adaptive PPIC multiuser detector based on PSO-MSE algorithms is studied. The performance evaluation of the proposed PSO-LMS scheme is provided by simulation results in Section IV. Finally, conclusions are given in Section V.

\section{SYSTEM MODEL}

We consider a CDMA communication system in which $K$ users are communicating simultaneously at the same rate over a common additive white Gaussian noise (AWGN) channel each with a binary phase-shift keying (BPSK) data modulation and its own PN code. In the reverse link of a synchronous CDMA system, the $k$ th user transmits the binary data signal $b_{k}(t)=\sum_{i=-\infty}^{\infty} b_{k, i} p_{T}(t-i T)$, where $b_{k, i} \in\{-$ $1,+1\}$ is the $i$ th data bit of the $k$ th user and $p_{T}(t)$ is a rectangular pulse with amplitude 1 and duration $T$. During the $i$ th bit, the $k$ th user's signal is spread by the signature waveform $a_{k}(t)=\sum_{i^{\prime}=-\infty}^{\infty} \sum_{j=1}^{N} a_{k, j} p_{T_{c}}\left[t-i^{\prime} T-(j-1) T_{c}\right]$, where $a_{k, j}=\{-1,+1\}$ is the $j$ th chip of the $k$ th user, and $p_{T_{c}}(t)$ is a rectangular chip pulse with duration $T_{c}$. The ratio of the bit duration $T$ to the chip duration $T_{c}$ is the processing gain $N=$ $T / T_{c}$.

For simplicity, the equivalent complex baseband representation for a synchronous CDMA system is considered in this paper. Thus, at the base station, the complex form of the received signal sampled at the chip rate after normalized chip-matched filtering shown in the receiver with the discrete-time representation for synchronous CDMA systems, can be written as

$$
\mathrm{r}(m)=\sum_{\lambda=1}^{L_{k}} \sum_{k=1}^{K} A_{k, \lambda, i} \cdot \mathbf{S}_{k}(m-\lambda+1)+\mathrm{n}(m),
$$

where $A_{k, \lambda, i}$ is the received signal amplitude sampled in the $i$ th bit for the $\lambda$ th path of $k$ th user and $\mathrm{n}(m)=n_{I}(m)+j n_{Q}(m)$ is a complex-valued AWGN with independent real and imaginary components and the two-sided PSD equals to $N_{o} / 2$. The maximum number of paths of the $k$ th user is denoted by $L_{k}$. At flat fading channel, there exists only one path, i.e., $L_{k}$ $=1$. When the frequency selective fading occurs, the number of paths is greater than 1, i.e., $L_{k}>1$. Moreover, the discretetime complex-value synchronous symbol is

$$
\mathbf{s}_{k}(m)=b_{k}(m) a_{k}(m) e^{j \phi_{k, i}},
$$

where $\phi_{k, i}$ are the received signal phase sampled at the $i$ th bit of the $k$ th user and the $a_{k}(m)=a_{k, j}, j=m \bmod N$ is the spreading chips.

In the Rayleigh flat fading channels, the first stage of the multistage PIC multiuser detector is a conventional CDMA receiver using a matched-filter as shown in Figure 1. The decision statistic for the $i$ th bit of the $k$ th user then become

$$
Z_{k, i}=\mathfrak{R}\left\{\frac{1}{N} \sum_{m=i N+1}^{(i+1) N} \mathrm{r}(m) \cdot a_{k}(m) e^{-j \phi_{k, i}}\right\} .
$$

However, over the frequency selective fading channels, to take the advantage of frequency diversity, the RAKE receiver is used [17]. In the RAKE receiver, the combining technique, referred to as the maximal ratio combining (MRC), maximize the instantaneous signal to interference and noise ratio (SINR) [17]. Therefore, with MRC, the decision statistic for the $i$ th bit of the $k$ th user is obtained as

$$
Z_{k, i}^{m r c}=\Re\left\{\frac{1}{L_{k} N} \sum_{\lambda=1}^{L_{k}} \sum_{m=i N+\lambda}^{(i+1) N+\lambda-1} \mathrm{r}(m) \cdot \hat{A}_{k, \lambda, i} \cdot a_{k}(m-\lambda+1) e^{-j \phi_{k, \lambda, i}}\right\} \cdot
$$

Then, the $i$ th data bit can be estimated by hard decision using the formula $\hat{b}_{k, i}=Z_{k, i}\left|Z_{k, i}\right|$.

In a multistage PPIC, the new received signal $r_{k}^{(s)}(m)$ for the $k$ th user after stages is given by

$$
\mathbf{r}_{k}^{(k)}(m)=\mathrm{r}(\mathrm{m})-\sum_{N=1 N=K}^{K} \lambda_{N}^{(k)}(m) \cdot \hat{A}_{K}(m) \cdot \hat{S}_{K}^{(k)}(m),
$$

where $\lambda_{K}^{(k)}(m)$ is the PCW at the $m$ th chip for the $k$ th user at stage $k$ and $0 \leq \lambda_{k}^{(k)}(m) \leq 1$.

Provided that the perfect knowledge of the entire user' amplitudes is available, from (4) and (5), it is evident that the performance of CPIC is heavily dependent on the accuracy of tentative data estimates.

\section{Multistage Adaptive Partial Parallel} INTERFERENCE CANCELLATION BASED ON LMS ALGORITHM

The LMS algorithm can adapt the optimum weight to perform an effective partial interference cancellation under light system load [10]. However, due to the slow convergence, the adaptation weight can not accurately estimate the amplitude of the desired signal when an incorrect bit-decision occurs. On the other hand, the statistics of adaptation-weight reveals that mean values are different between the correct-decision and incorrect-decision bits as described in [10]. Therefore, prior to a full PIC, the bitinversion procedure based on LMS algorithms (BI-LMS) is proposed to reverse part of error bits as decribed in [8]. To 
study the bit reliability of the bit-inversion procedure, we investigate the BER for APPIC scheme by computer simulation with the constant PCWs $0.75 \leq w_{k} \leq 1$ under perfect power control situations over Rayleigh flat fading channels. In all simulations, the Gold codes [10] are generated for each user and used to spread his or her random data bits with the processing gain 31 for the synchronous CDMA systems. The results of these spreading operations are multiplied by the carrier phases following which Gaussian noise samples are added to the combined received signal with one sample per chip time. We assumed that the time and phase synchronizations are perfect. The SNR and amplitudes of users in channel estimations are also assumed to be perfectly estimated.

The functional block diagram of adaptation PCW based on the LMS algorithm in the PIC scheme (LMS-PIC) for synchronous CDMA systems is shown in Fig 1. During a bit interval, at the $m$ th chip, the estimation error in matrix form obtained as

$$
\begin{aligned}
\mathbf{e}(m) & =\mathbf{r}(m)-\sum_{k=1}^{K} \lambda_{k}(m) \hat{b}_{k} a_{k}(m) e^{i \phi_{k}} \\
= & \mathrm{r}(m)-\lambda^{T}(m) \hat{\mathbf{S}}(m),
\end{aligned}
$$

where $\lambda(m)=\left[\lambda_{1}(m) \lambda_{2}(m) \cdots \lambda_{K}(m)\right]^{T}$ is the adaptationweight vector for all users, and $\mathbf{S}(m)=\left[\begin{array}{lll}S_{1}(m) & S_{2}(m) \cdots S_{K}(m)\end{array}\right]^{T}$ is the estimated signal vector of users, which can be expressed by $\hat{\mathbf{S}}(m)=\mathbf{a}(m) \cdot \hat{\mathbf{b}}$, where $\hat{\mathbf{b}}=\left[\hat{b}_{1}, \hat{b}_{2}, \cdots, \hat{b}_{K}\right]^{T}$, $\mathbf{a}(\mathrm{m})=\operatorname{Diag}\left[a_{1}(m) e^{i \phi 1}, a_{2}(m) e^{i \phi 2}, \cdots, a_{k}(m) e^{i \phi K}\right]$ and $\mathbf{a}(m)$ is a $K \times K$ matrix. In this work, we motivates us to find the optimal weights for MAI cancellation. Suppose that the power levels of all users are known to the receiver at the base station.

The optimal cost function is defined as the expectation of the squared distance between the received signal and adaptation-weighted sum of the estimates all user signals [8] i.e..

$$
J(m)=E\left[\left|r(m)-\lambda^{T}(m) \hat{\mathbf{S}}(m)\right|^{2}\right] .
$$

We attempt to minimize the MSE with a set of the optimal adaptation-weights $\{\lambda\}$ [15-16].

Since the LMS algorithm is based on the MSE criteria, the cost function given in (7) is modified as follows:

$$
\min _{\lambda^{(k)}} E\left[\left|r(m)-\hat{r}^{(k)}(m)\right|^{2}\right] \text {. }
$$

where $\hat{r}^{(k)}(m)=\boldsymbol{\lambda}^{T}(m) \hat{\mathbf{S}}(m)$.

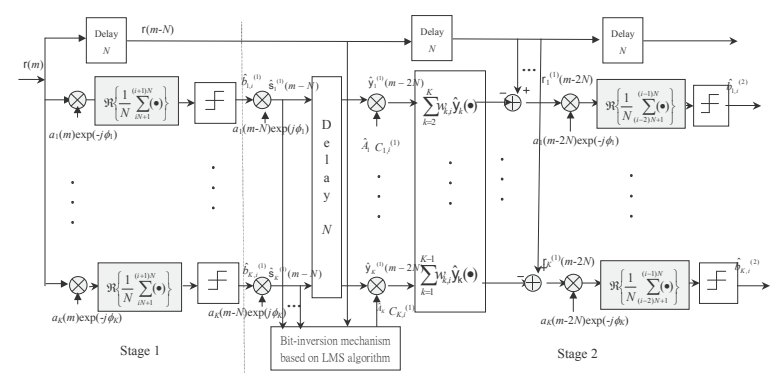

Fig. 1. The cancellation weight adaptor based on PSO-LMS scheme for synchronous CDMA systems.

The optimal partial cancellation weight is an NPcomplete combinatorial optimization problem and traditional optimization algorithms are inefficient for its solution. PSO was first proposed in by R. C. Eberhart et al. [19-20] as an approach to achieve artificial intelligence. It has been recently applied with success to many numerical and combinatorial optimization problems [21]. Unlike GAs, PSO has no evolution operators such as crossover and mutation. It has memory knowledge of good solutions is retained by all particles, whereas in GA, previous knowledge of the problem is destroyed once the population changes. PSO exhibits constructive cooperation between particles, since particles in the swarm share information between them. This technique combines social psychology principles in sociocognition human agents with evolutionary computation. PSO is usually characterized as a simple concept, which is easy to implement and computationally efficient. Unlike other heuristic techniques, PSO has a flexible and well-balanced mechanism to enhance the global and local exploration abilities.

The LMS algorithm can adapt the PCW to perform an effective partial interference cancellation under light system load [8]. However, due to the slow convergence, the adaptation-weight can not accurately estimate the amplitude of the desired signal when an incorrect bit-decision occurs. On the other hand, the statistics of adaptation-weight reveals that mean values are different between the correct-decision and incorrect-decision bits as described in [10]. Therefore, prior to a full PIC, the bit-inversion procedure based on LMS algorithms (BI-LMS) is proposed to reverse part of error bits as decribed in [10].

Since the structure of system model is known in advance, the basic principle of parameter estimation is to compare the output of ture system and the estimated model under the same inputs, and to adjust the parameters $\lambda(m)=\left[\lambda_{1}(m) \lambda_{2}(m) \cdots \lambda_{K}(m)\right]^{T}$ for minimizing a predefined error function, e.g., the following mean square error function as Eq.(8)

$$
\min J(m)=E\left[\left|r(m)-\lambda^{T}(m) \hat{\mathbf{S}}(m)\right|^{2}\right]=E\left[\left|r(m)-\hat{r}^{(k)}(m)\right|^{2}\right] \quad,
$$

where $\hat{r}^{(k)}(m)$ is the estimate of the received signal in the $k$ th stage. 


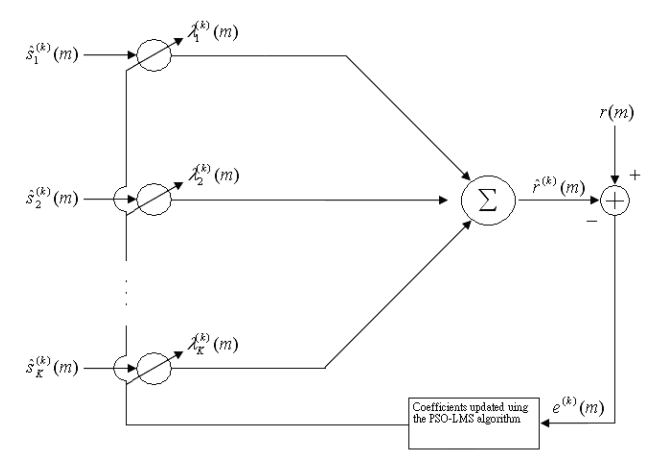

Fig. 2. Weight updating in the adaptive multistage PIC in the AWGN channel

Obviously, parameter estimation essentially is a multidimensional numerical optimization problem, where decision variables are parameters $\lambda(m)=\left[\lambda_{1}(m) \lambda_{2}(m) \cdots \lambda_{K}(m)\right]^{T}$, objective function is $F$, and the optimization algorithm is used to adjust model parameters. Due to the non-linear property of complex systems, it is hard to estimate all parameters precisely. Moreover, from the viewpoint of optimization, there may be multiple local optima on the corresponding, are easy to be trapped in local optimal. In our previous work [23], GA and PSO strategies have been proposed, whose performances are much better than those of traditional methods and pure single approach. Compared with GA and PSO, PSO has some superiority as mentioned before and is easy to implement as well. The PSO optimization procedure resembling the social behavior of a swarm of bees, searching for the location with the most flowers in a field. It is based on a population of particles that fly in the solution space with velocity that is dynamically adjusted based on its own flying experience and that of the best among the swarm. Fig. 3 depicts the flow chart of a PSO algorithm. Each potential solution in the PSO process is represented as a particle with a position vector denoted as $\lambda$ (referred to as adaptation-weights) and a moving velocity denoted as $\mathbf{v}$, respectively. Thus for an $K$-dimensional optimization, the position and velocity of the $i$ th particle is represented as $\lambda_{i}=\left(\lambda_{i, 1}, \lambda_{i, 2}, \ldots, \lambda_{i, N}\right)$ and $v_{i}=\left(v_{i, 1}, v_{i, 2}, \ldots, v_{i, N}\right)$. Every particle of $\lambda_{i}$ keeps track of its position, which is uniformly spread for each of the $N$, dimensions in the solution space, bounded by $\left[\lambda_{i, n}^{\min }, \lambda_{i, n}^{\max }\right]$ to prevent particles from flying out of the physically meaningful solution space. Here, $n$ represents the $n$th dimension. In contrast to a GA, the PSO also begins by generating a population of particles at random. The randomness enables the exploration of a broad population of possible solutions in the entire search space. At every time step, an associated value for each particle is evaluated according to with a function called the cost function, which is critically defined and configured by considering the search objective with $\lambda_{i}$ as input. The value normally called the cost indicates the goodness of the solution. However, a practical implementation has not only one possible fitness function that can reflect the design objective. A function that can best represent the relative importance of each goal can often be obtained through trial and error.

The position of the individual best fitness achieved by particle $i$ has achieved so far. Meanwhile, that of the highest fitness which has been obtained among all the particles in the population so far, are called the personal best (denoted as $\lambda_{i}^{\text {best }}$ ) and the global best (denoted as $\lambda^{\text {best }}$ ), respectively, and both are stored to generate the new velocity of particle $i$. Each particle adjusts its velocity during the process according to its own experience and the position of the best of all particles to move toward the best solution. Meanwhile, a condition is also set during the following step, controlling the algorithm when it stops by either setting it to obtain an acceptable target solution or to run for a set maximum number of search iterations. If the algorithm does not step, after a time step, $\Delta t$, then the new velocity $v_{i}(t+\Delta t)$ for particle $i$ is updated by

$$
\begin{aligned}
& v_{i}(t+\Delta t)=w \cdot v_{i}(t)+c_{1} * \operatorname{rand}() *\left(\lambda_{i}^{\text {best }}(t)-\lambda_{i}(t)\right) \\
& +c_{2} * \operatorname{rand}() *\left(\lambda^{\text {best }}(t)-\lambda_{i}(t)\right)
\end{aligned}
$$

where $v_{i}(\mathrm{t})$ denotes the old velocity of the particle $i$ at time $t$. Significantly, in the real implementation of a PSO time $t$ and $t^{+} \Delta t$ generally represent the current and next iterations, respectively, therefore, so $\Delta t$ in (9) and (11) is always omitted (i.e. its value is assumed to be to 1 ) in most literature. This equation reveals that the new velocity is related to the old velocity weighted by $w$, and is also associated with the position of the particle itself and of the global best one by factors $c_{1}$ and $c_{2}$ respectively. The parameters $c_{1}$ and $c_{2}$ are set to constant values, and are therefore known as the cognitive and social rates, respectively, since they represent the weighting of the acceleration terms pulling the individual particle toward the personal and global best positions. The inertia weight $w$ in (10) is utilized to manipulate the impact of the previous history of velocities on the current velocity [19]. Population based optimization methods should generally encourage individuals to wander through the entire search space, without clustering around the local optima, during the early stage of the optimization. Conversely, during later stages, convergence toward the global optima should be enhanced to discover the optimum solution efficiently. Therefore, $w$ resolves the tradeoff between the global and local exploration ability of the swarm. A large inertia weight promotes global exploration, while a small one promotes local exploration, i.e., fine-tunes the current search area. An appropriate value for $w$ provides the desired balance between the global and local exploration ability of the swarm, and consequently enhances the effectiveness of the algorithm. Typical implementations of the PSO adapt the value of $w$ during the training stage, e.g., linearly decreasing it from 0.9 to near 0.4 over the execution. Experimental results suggest that it is preferable to initialize the inertia weight should 
ideally be initialized to a large value, giving priority to global exploration of the search space and should be linearly decreasing to obtain refine the solutions [20-21]. In this work, the inertia weight $w$ is set based on the following equation

$$
w=w_{\max }-\left(\left(w_{\max }-w_{\min }\right) /\left(\text { iter }_{\max }\right)\right) \times \text { iter },
$$

where iter $_{\max }$ is the maximum number of iterations; iter is the current iteration number; $w_{\max }$ is the initial weight, and $w_{\min }$ is the final weight. To simulate the small unpredictable component of natural swarm behavior, two random functions rand() are applied to provide independently uniformly distributed sets of numbers in the rang $[0,1]$ to vary stochastically the relative pull of the personal and global best particles. The new position for particle $i$ is determined form the updated velocities the following equation:

$$
\lambda_{i}(t+\Delta t)=\lambda_{i}(t)+\Delta t * v_{i}(t+\Delta t)
$$

The populations of particles are then moved according to the new velocities and locations obtained with (9) and (11), and tend to cluster together from different directions. The evaluation of each associate fitness of the new population of particles is thus repeated. The algorithm runs iteratively through these processes until it stops. Each particle is an $m$ dimensional real-valued vector, where $m$ is the number of optimized parameters. Therefore, each optimized parameter represents a dimension of the problem space.

The proposed PSO- LMS algorithm based on PIC multiuser detector for a $K$-user synchronous CDMA system can be outlined as follows:

Step 1: Solution Representation: The multiuser detection can be regarded as an optimization problem that finds the most likely combination of optimal weights $\lambda_{O M P, p}=$ $\left[\lambda_{1, p}, \lambda_{2, p}, \cdots, \lambda_{K, p}\right]$ is $0 \leq \lambda_{K, p} \leq 1$.

Step 2: Initialization: Initialize a population of particles with random positions and velocities, where each particle contains $\mathrm{K}$ variable.

Step 3: Evaluate the fitness values of all particles, let pbest of each particle and its objective value equal to its current position and objective value, and let gbest and its objective value equal to the position and objective value of the best initial particle.

Step 4: Update the velocity and position of each particle according to Eqs. (9) and (11).

Step 5: Evaluate the objective values of all particles according to Eqs. (8).

Step 6: for each particle, compare its current objective value with the object value of its pbest. If current value is better, then update pest and its object value with the current position and objective value. Furthermore, determine the best particle of current warm with the best objective values. If the objective value is better than the object value of gbest, then update gbest and its objective value with the position and objective value of the current best particle.

Step 7: Termination criteria: If a predefined stopping criterion is met, then output gbest and its objective value; otherwise go back to Step 4.

\section{Simulation Results}

In this section we analyze the performance of the aforementioned PSO-LMS as multiuser detectors in CDMA system. To compare the performance of the LMS based PIC schemes, we perform the simuation in the near-far situations over flat fading channels as follows.

The relationship between the PCW and the effective number of users and $S N R$ of the desired user has been described in [11]. Therefore, we can observe that the higher the SNR is, the larger the PCW is, and the less the effective number of user is, the larger the PCW is, too. Because of the high reliability of the bit-inversion procedure, in stage 3 , the full PIC is used to indeed fully cancel the MAI instead of PPIC. Thus, we can construct a PSO-LMS algorithm based on multistage PIC for multiuser detection as shown in Fig. 4.

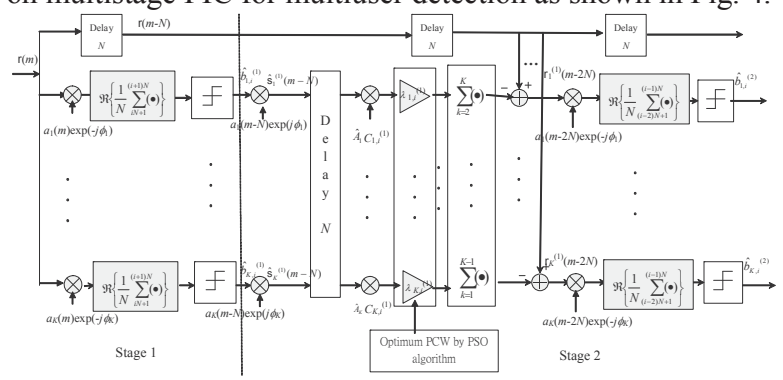

Fig. 4. Multistage adaptive partial PIC scheme based on PSO-LMS algorithm for synchronous CDMA systems.

We investigate the BER for PSO-LMS algorithm and bit-inversion based multistage PIC scheme by computer simulation with the PCWs $0 \leq \lambda \leq 1$ under perfect power control situations over Rayleigh flat fading channels. In all simulations, the Gold codes [12] are generated for each user and used to spread his or her random data bits with the processing gain 31 for the synchronous CDMA systems. The results of these spreading operations are multiplied by the carrier phases following which Gaussian noise samples are added to the combined received signal with one sample per chip time. We assumed that the time and phase synchronizations are perfect. The SNR and amplitudes of users in channel estimations are also assumed to be perfectly estimated.

Fig. 5 depicts the comparison of BER vs. SNR of the low power users for the bit-inversion based PIC detectors with the number of users $K=24$ and $N F R=6 \mathrm{~dB}$. Moreover, Fig. 6 shows the results of BER vs. number of users with the $\mathrm{NFR}=6 \mathrm{~dB}$ and $S N R=6 \mathrm{~dB}$ for the low power users for the bit-inversion based PIC detectors. However, in the results of Figs. 5 and 6, we can see that the PSO-LMS improves slightly the performance of the AFPIC. All methods provide a better performance than the conventional receivers. Among the second stage schemes, the PSO-LMS and the AFPIC methods have the best performance. For example, to maintain a BER of 0.01, the BI-PIC and the AFPIC improve the capacity to 14 and 28 users, respectively, while the PSOLMS has a comparable performance and can accommodate 30 users. By increasing number of users of the PSO-LMS technique is further improved. 


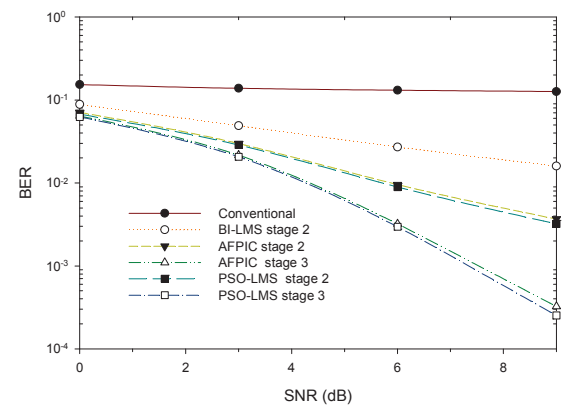

Fig. 5. BER vs. SNR of the low power users for the multistage bitinversion based PIC detectors with the number of users $K=24$ and the $N F R=6 \mathrm{~dB}$.

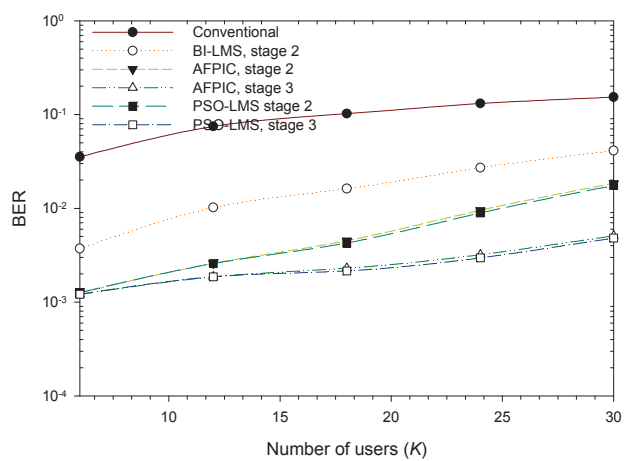

Fig. 6. BER vs. number of users for the multistage LMS based PIC detectors with the $N F R=6 \mathrm{~dB}$ and $S N R=6 \mathrm{~dB}$ for the low power users.

Furthermore, to show the performance improvements on the frequency-selective fading channels, we perform simuation over a two-path frequency-selective fading channel as follows. The two-path fading channel is modeled with equal average power and the maximum number of paths of the $k$ th user, $L_{k}=2$ in (1). Fig. 7 shows the BER vs. SNR curves for bit-inversion based PIC multiuser detectors over a two-path frequency selective fading channel with $K=20$. Moreover, Fig. 8 shows the BER vs. number of users curves for bit-inversion based PIC multiuser detectors over a twopath frequency selective fading channel with $S N R=12 \mathrm{~dB}$. In Fig. 7 and Fig. 8, the PSO-LMS slightly outperforms the AFPIC. Therefore, it should be noted that after the proposed bit-inversion procedure, the most part of user bits with high received power has been inverted; the AFPIC scheme thus cancels nearly all interference.

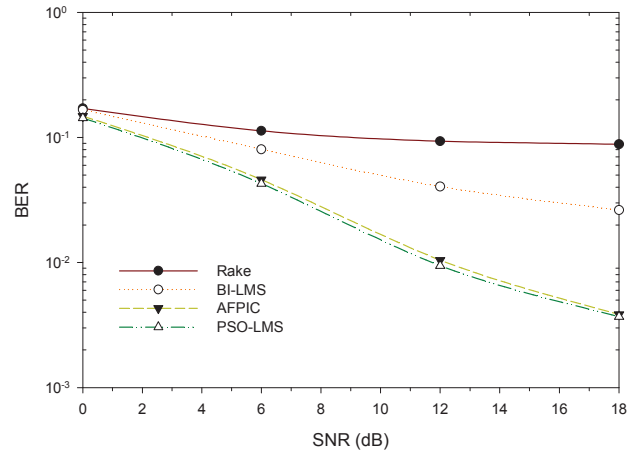

Fig. 7. BER vs. SNR for the two-stage bit-inversion based PIC multiuser detectors over a two-path frequency selective fading channel with $K=20$.

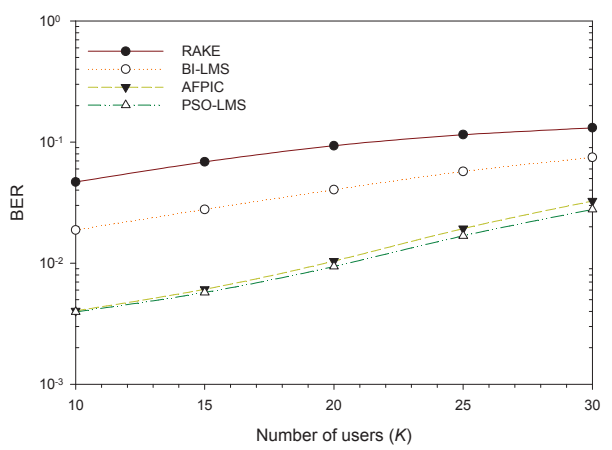

Fig. 8. BER vs. number of users for the two-stage bit-inversion based PIC multiuser detectors over a two-path frequency selective fading channel with $S N R=12 \mathrm{~dB}$

\section{CONCLUSIONS}

In this paper, we apply PSO-MSE algorithm based multistage PPIC detection scheme solutions for optimum PCWs in AWGN and multipath fading channels. Our analysis results in significant reduction in the receiver complexity. The BI-LMS scheme corrects most part of the bit-decision errors in the previous stage with an amplitudedependent threshold. Moreover, after the bit-inversion, an adaptive PPIC can alleviate the excess cancellation errors of the full PIC at heavy system load. Simulation results show that some performance improvement has been achieved.

\section{REFERENCES}


[1] K. S. Gilhousen, I. M. Jacobs, R. Padavani, A. J. Viterbi, L. A Weaver and C. E. Wheatley, "On the capacity of a cellular CDMA system," IEEE Trans. Vehicular Tech., vol. 40, no. 2, pp. 303-312, May 1991.

[2] Verdu S. "Minimum probability of error for asynchronous Gaussian multiple access channel," IEEE Trans. Inform. Theory, Vol. IT-32, pp. 85-96, Jan. 1986.

[3] Markku J. Junti and Behnaam Aazhang, "Finite memory-length linear multiuser detection for asynchronous CDMA communication," IEEE Trans. Commun., vol. 45, pp. 611-622, May. 1997.

[4] S. Verdú, Multiuser Detection, Cambridge University Press, 1998.

[5] D. Guo, L. K. Rasmussen, S. Sun and T. J. Lim, "A matrix algebraic approach to linear parallel interference cancellation in CDMA," IEEE Trans. Commun., vol.48, pp. 152-161, Jan 2000.

[6] M. K. Varanasi and B. Aazhang, "Multistage detection in asynchronous code division multiple-access communications," IEEE Trans. Commun., vol. COM-38, no. 4, pp. 509-519, April 1990.

[7] D. Divsalar, M. K. Simon and D. Raphaeli, "Improved parallel interference cancellation for CDMA," IEEE Trans. Commun., vol. COM-46, no. 2, pp. 258-268, Feb. 1998.

[8] G. Xue, J. Weng, Tho Le-Ngoc, and S. Tahar, "Adaptive multistage parallel interference cancellation for CDMA," IEEE J. Select. Areas Commun., vol. 17, no. 10, pp. 1815-1827, Oct. 1999.

[9] Y. F. Huang and J. H. Wen, "An Adaptive Multistage Parallel Interference Canceller for CDMA Systems Over Rayleigh Fading Channels," Proc. of IEEE GLOBECOM 2002, Taipei, Taiwan, Nov. 17-21, 2002.

[10] Y. F. Huang and J. H. Wen, "An Analysis on Partial PIC Multi-User Detection with LMS Algorithms for CDMA," Proc. of IEEE PIMRC 2003, Peijin, China, pp. 17-21, Sept. 7-10, 2003.

[11] J. H. Wen and Y. F. Huang, "Fuzzy-based adaptive partial parallel interference canceller for CDMA communication systems over fading channels," IEE Proceedings-Communications, vol. 149, no. 2, pp. 111-116, April 2002.

[12] Fawzy Aan, Fayez R. M. "Optimization of partial parallel interference cancellation factor in CDMA system." In Proc. IEEE International Conference on Systems, Man, and Cybernetics, (4), pp. 2375-2380, 2000.
[13] Chen-Chu Hsu and Yumin Lee, "Multistage weighted interference cancellation with receiver diversity for DS-CDMA," IEEE Trans. on Signal Proce. Vol. 52, pp. 558-565, Feb. 2004.

[14] Ana M. Barbancho, Lorenzo J. Tardon, and Isabel Barbancho, "Analytical performance analysis of the linear multistage partial PIC receiver for DS-CDMA systems," IEEE Trans. On Comm. Vol. 53, pp. 2006-2010, DEC. 2005.

[15] Yu-Tao Hsieh and Wen-Rong Wu, "Optimal two-stage decoupled partial PIC receiver for multiuser detection," IEEE Trans. On Wireless Commu. Vol. 4, pp. 112-127, Jan. 2005

[16] Seong Rang Kim, In-Kyeong Choi, Sugbong Kang and Jeong Goo Lee, "Adaptive weighted parallel interference cancellation for CDMA systems," Electronics Letters, vol. 34, pp. 2085-2086, October 1998.

[17] Proakis J. Digital communications. Englewood Cliffs, NJ: PrenticeHall, 2001.

[18] R. C. Eberhart and J. Kennedy," A new optimizer using particle swarm theory," in Proc. $6^{\text {th }}$ Symp. Micro Machine and Human Science, Nagoya, Japan, pp. 39-43. 1995.

[19] R. C. Eberhart and J. Kennedy, "Particle swarm optimization," in Proc. IEEE Conf. Neural Networks IV, Piscataway, NJ, PP. 19421948, 1995.

[20] Asanga Ratnaweera, Saman K. Halgamuge and Harry C. Watson.,"Self-organizing hierarchical particle swarm optimizer with time-varting accleration coefficients," IEEE Trans. Evol. Comput. Vo.8 (2004), 240-255.

[21] S. L. Ho, Shiyou Yang, Guangzheng Ni, Edward W. C. Lo, and H. C. Wong, "Aparticle swarm optimization-based method for multiobject design optimizations," IEEE Trans. on Magnetic, vol. 41, pp. 17561759, May, 2005.

[22] Maurice Clerc and James Kennedy, "The particle swarm-explosion, stability, and convergence in a multidimensional complex space," IEEE Trans. on Evolutionary Computation, vol. 6, pp. 58-73, Feb. 2002.

[23] H. L. Hung, Y. F. Huang and J. H. Wen.: A Particle Swarm Optimization based Multiuser Detector for DS-CDMA Communication Systems. Process. of IEEE Conf. on SMC (2006), pp.1956-1961.G. Eason, B. Noble, and I. N. Sneddon, "On certain integrals of Lipschitz-Hankel type involving products of Bessel functions," Phil. Trans. Roy. Soc. London, vol. A247, pp. 529-551, April 1955. (references) 